\title{
Effects of heterogeneous surface boundary conditions on parameterized oceanic deep convection
}

\author{
Martin Losch ${ }^{\mathrm{a}, 1}$, Sandra Herlufsen ${ }^{\mathrm{b}}$, Ralph Timmermann ${ }^{\mathrm{a}}$ \\ a Alfred-Wegener-Institut für Polar- und Meeresforschung, Postfach 120161, 27515 Bremerhaven, Germany \\ ${ }^{\mathrm{b}}$ Universität Bremen, Germany
}

\begin{abstract}
Vertical mixing and deep convection are routinely parameterized in basin-scale and global ocean general circulation models. These parameterizations are designed to work with homogeneous surface boundary conditions for an individual grid cell. A partial ice cover, however, yields heterogeneous fluxes of buoyancy that are not resolved by the computational grid. The effects of such heterogeneous surface boundary conditions are explored by comparing coarse resolution models with three common parameterizations for mixing and deep convection with large eddy simulations (LES) of free convection in idealized scenarios. Generally, models with parameterized convection reproduce the temperature profiles of the LES reference accurately when the surface boundary conditions are resolved by the grid. Significant biases are introduced when the surface boundary conditions are not resolved and buoyancy fluxes are averaged horizontally. These biases imply that mixing depths may locally be too shallow in large scale simulations without proper handling of heterogeneous boundary conditions during convective events; also the grid-cell averaged density may be vertically homogenized within the shallow boundary layer. Adaptation of present mixing schemes may overcome these spurious effects of horizontally averaging the surface buoyancy fluxes.
\end{abstract}

Key words: NUMERICAL OCEAN MODELING, DEEP CONVECTION, HETEROGENEOUS BOUNDARY CONDITIONS, SEA-ICE

\section{Introduction}

Turbulent deep convection in the ocean is driven by buoyancy loss at the surface. After a preconditioning phase, rapid surface cooling at the polar convection sites, for example in the Labrador and Greenland Seas, leads to violent and deep-reaching motion that mixes surface waters to great depth, setting and maintaining the properties of the abyss (Marshall and Schott, 1999). In other regions, for example the Weddell Sea, sea-ice formation increases the surface salin-

\footnotetext{
1 corresponding author, email: mlosch@awi-bremerhaven.de, ph: ++49 (471) 4831-1872, fax: ++49 (471) 4831-1797
}

ity and thus destabilizes the water column (Mosby, 1934, Foster, 1972). Numerical ocean general circulation models (OGCMs), in particular those used in climate studies, are still too coarse to resolve convection explicitly, and suffer further from a common lack of non-hydrostatic dynamics which is important on convective scales. Still the overall effect of deep mixing, namely the formation of deep water mass characteristics, is necessary for these models to maintain realistic abyssal water mass properties and circulation.

Therefore, vertical mixing and convection in OGCMs generally is parameterized. Parameterizations include bulk mixed-layer models (e.g., Krauss and Turner, 1967), first order (e.g., Pacanowski and 
Philander, 1981, Large et al., 1994) and second order (e.g., Mellor and Yamada, 1982, Gaspar et al., 1990) turbulence closure models. Deep convection is usually modeled by even cruder methods, for example the socalled "convective adjustment" or increased vertical diffusivities in the case of unstable stratification (e.g., Haidvogel and Beckmann, 1999).

In polar regions, where important oceanic convection sites are located, the seasonal ice cover is a major factor determining the oceanic surface fluxes of buoyancy, insulating the ocean from the atmosphere in some places and exposing it to cold air and precipitation in others. Sea-ice formation and melting can also modify the stratification by brine rejection and fresh water release, respectively. Usually, coupled OGCMs and sea-ice models are too coarse to resolve the spatial and temporal distribution of ice floes. Instead, the seaice models describe sea-ice concentrations, which give the areal fraction of sea-ice within each grid box. For an OGCM this means that grid cells can be partially covered with sea-ice so that the surface boundary conditions for these grid cells are formally heterogeneous. Usually, the total surface buoyancy flux for such cells is the average over the ice-covered and open-water fraction of the cell (e.g., Timmermann et al., 2002). Therefore, high buoyancy fluxes out of the ocean over open water or increasing salinity by ice formation, which potentially could lead to an unstable water column and deep mixing, contribute only in part to the overall buoyancy flux of the grid cell, thereby reducing the potential for localized deep mixing.

In climate OGCMs, the situation may become even more difficult, because deep convection and mixing in the ocean model is parameterized. In addition to the grid-cell averaged buoyancy flux, the description of the vertical mixing is also horizontally averaged over the grid cell, thereby further diluting the impact of a localized buoyancy loss at the surface.

In this note, we present the results of a systematic study that aims to assess the effects of heterogeneous surface boundary conditions on mixing and mixing parameterizations. Due to the sparsity of ocean data on turbulent convection we choose a large eddy simulation (LES) approach, where a numerical nonhydrostatic ocean model (the MITgcm, Marshall et al., 1997) is integrated at very high resolution to simulate a convection event. The model data is used for benchmarking subsequent coarse resolution, hydro- static cousin-experiments, also with the MITgcm, in which the convection is parameterized. Two situations are of interest: a. the horizontal grid resolves the icecover or surface buoyancy flux heterogeneity and all errors can be attributed to the mixing scheme; b. the horizontal grid is too coarse and both averaged buoyancy flux and mixing scheme contribute to the deviation from the reference. The convection parameterizations that we test here are convective adjustment, as implemented in the MITgcm, "implicit diffusion", and the KPP mixed layer model of Large et al. (1994).

\section{Numerical Experiments}

\subsection{LES Reference}

The M.I.T. General Circulation Model (MITgcm) is a general purpose grid-point algorithm that solves the Boussinesq form of the Navier-Stokes equations for an incompressible fluid, hydrostatic or fully nonhydrostatic, with a spatial finite-volume discretization on a curvilinear computational grid. The model algorithm is described in Marshall et al. (1997), for online documentation and access to the model code, see MITgcm Group (2002).

For computational efficiency we choose a domain that represents a horizontal section through the ocean in the $x$-z-plane. The domain is doubly periodic in the horizontal, $15 \mathrm{~km}$ wide, and $1 \mathrm{~km}$ deep; the grid spacing is $10 \mathrm{~m}$ in both the horizontal and the vertical direction. Acceleration due to gravity is $g=9.81 \mathrm{~m} \mathrm{~s}^{-2}$. A Coriolis parameter of $f=1.4 \times 10^{-4} \mathrm{~s}^{-1}$ leads to a regime of convection that is affected by rotation.

The sub-grid-scale processes are approximated with harmonic dissipation of momentum with variable viscosity following Leith (1968b,a). The tracer advection scheme is a third-order flux limited scheme (Hundsdorfer and Trompert, 1994, Hundsdorfer et al., 1995) that is unconditionally stable and does not require additional diffusion.

In all experiments, we assume a partial ice cover that insulates the ocean from the atmosphere. Only the central $5 \mathrm{~km}$ of the domain have open water, where, in all experiments, a constant surface heat flux $Q_{\text {net }}=$ $200 \mathrm{~W} \mathrm{~m}^{-2}$ out of the ocean is applied over the time of the integration. The value is chosen to represent 
typical buoyancy loss in the Labrador Sea (Marshall and Schott, 1999) in April. The ocean is governed by a linear equation of state

$$
\rho=\rho_{0}\left[1-\alpha\left(\theta-\theta_{0}\right)\right],
$$

where $\alpha=2 \times 10^{-4} \mathrm{~K}^{-1}$ is the coefficient of thermal expansion of water, and $\rho_{0}=999.8 \mathrm{~kg} \mathrm{~m}^{3}$ and $\theta_{0}=1{ }^{\circ} \mathrm{C}$ are constant reference density and temperature, respectively. Note that the simulations presented here do not contain any haline effects. In particular, nonlinear processes such as cabbeling are deliberately excluded in this study to isolate the effect of horizontal resolution. The initial temperature field is perturbed with random noise of small amplitude $\left(0.01{ }^{\circ} \mathrm{C}\right)$ to start the convection. The model is integrated for 96 hours. After 96 hours of integration "lateral exchange" between the convection site and the ambient fluid through advective processes (Marshall and Schott, 1999) is assumed to dominate. The lateral exchange cannot be represented properly by our two-dimensional configuration and the integration is stopped. During convection vertical velocities reach $\mathrm{O}(10 \mathrm{~cm} / \mathrm{s})$ so that the time for a water parcel to travel from the surface to the bottom is of the order of a few hours. The moments of vertical velocity scale with the surface heat flux as $(Q / f)^{(1 / 2)}$ as predicted for open ocean free convection (Golitsyn, 1980, Fernando et al., 1991, Jones and Marshall, 1993).

\subsection{Coarse Resolution Experiments}

The computational domain of the reference simulation is now spanned with coarser grids to simulate the resolution of a basin-scale OGCM. For the horizontal grid spacing $\Delta x$ we choose two different scenarios: 3 cells of length $5 \mathrm{~km}$ and 1 cell of length $15 \mathrm{~km}$. With the $5 \mathrm{~km}$-resolution the spatial distribution of the surface buoyancy loss is just resolved, and $Q_{\text {net }}$ is applied only at the central grid cell. For the $15 \mathrm{~km}$-grid cell, the surface boundary conditions are heterogeneous, and an average heat flux $\left\langle Q_{\text {net }}\right\rangle=\frac{1}{3} Q_{\text {net }}$ has to be applied.

In these hydrostatic simulations two different vertical resolutions are used: a small vertical grid spacing of $10 \mathrm{~m}$ to exclude effects of a different vertical resolution, and a coarser vertical resolution which was chosen to represent typical grids in OGCMs. In the latter case the vertical grid spacing increases from $10 \mathrm{~m}$ near the surface to $100 \mathrm{~m}$ at $1000 \mathrm{~m}$ depth. The coarse hydrostatic models are run with a 1 hour time step that is typical of a coarse, large-scale OGCM.

Three different vertical mixing schemes are used in the hydrostatic simulations:

convective adjustment (CA) In the present implementation of CA in the $\mathrm{MITgcm}$, the algorithm starts at the sea surface, compares the densities of a vertical pair of grid cells referenced to the same depth, and, if the upper cell is denser than the lower cell (static instability), replaces the densities by an average density. The next pair consists of the previously lower cell and the next cell below. This algorithm is slower than mixing the entire water column instantly down to the last unstable point (e.g., Madec et al., 1991), and surface forced instabilities are not necessarily removed within one time step and one vertical "sweep".

implicit diffusion (ID) Here, the vertical diffusivity is increased to a value of $10 \mathrm{~m}^{2} \mathrm{~s}^{-1}$ over the statically unstable part of the water column. Because of the high diffusivity, the vertical diffusion then needs to be treated implicitly, hence the name.

KPP (Large et al., 1994) The more sophisticated Kprofile parameterization is a first order turbulent closure scheme which models higher-order turbulent moments by a counter-gradient flux. For details the reader is referred to Large et al. (1994).

\section{Results}

\subsection{Unstratified conditions}

In the first experiment the initial stratification is neutral so that vigorous convection sets in immediately in the LES reference solution. Four snapshots of the temperature distribution after 24, 48, 72, and 96 hours of surface cooling are shown in Fig. 1. In Fig. 2 we compare the different parameterizations of the convection by means of horizontally averaged temperature profiles after 24, 48, 72, and 96 hours of continuous surface buoyancy loss. In the LES reference solution (solid line), convective plumes penetrate to the bottom of the domain and establish a stable stratification with increasingly cold (dense) water towards the bottom (see also Fig. 1). Only the KPP mixing scheme 


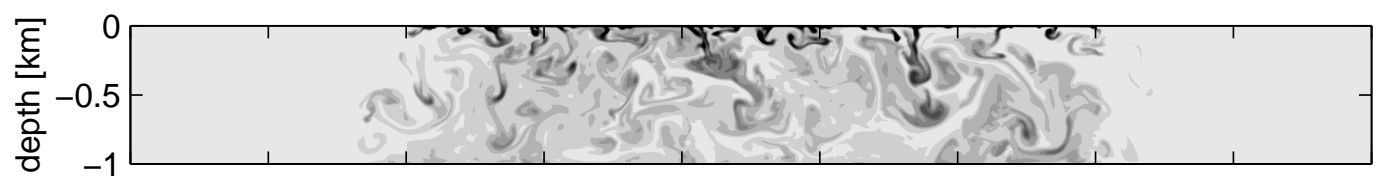

$48 \mathrm{~h}$

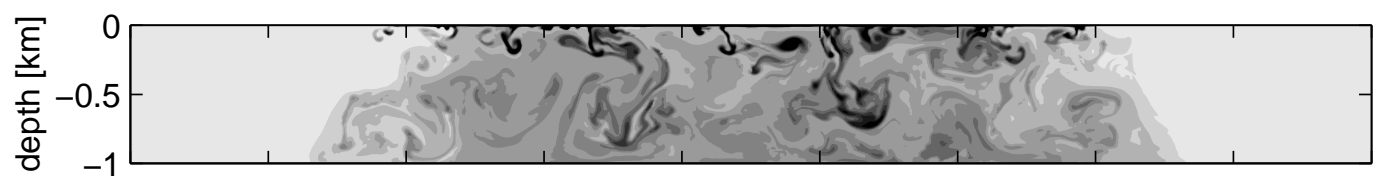

$72 \mathrm{~h}$
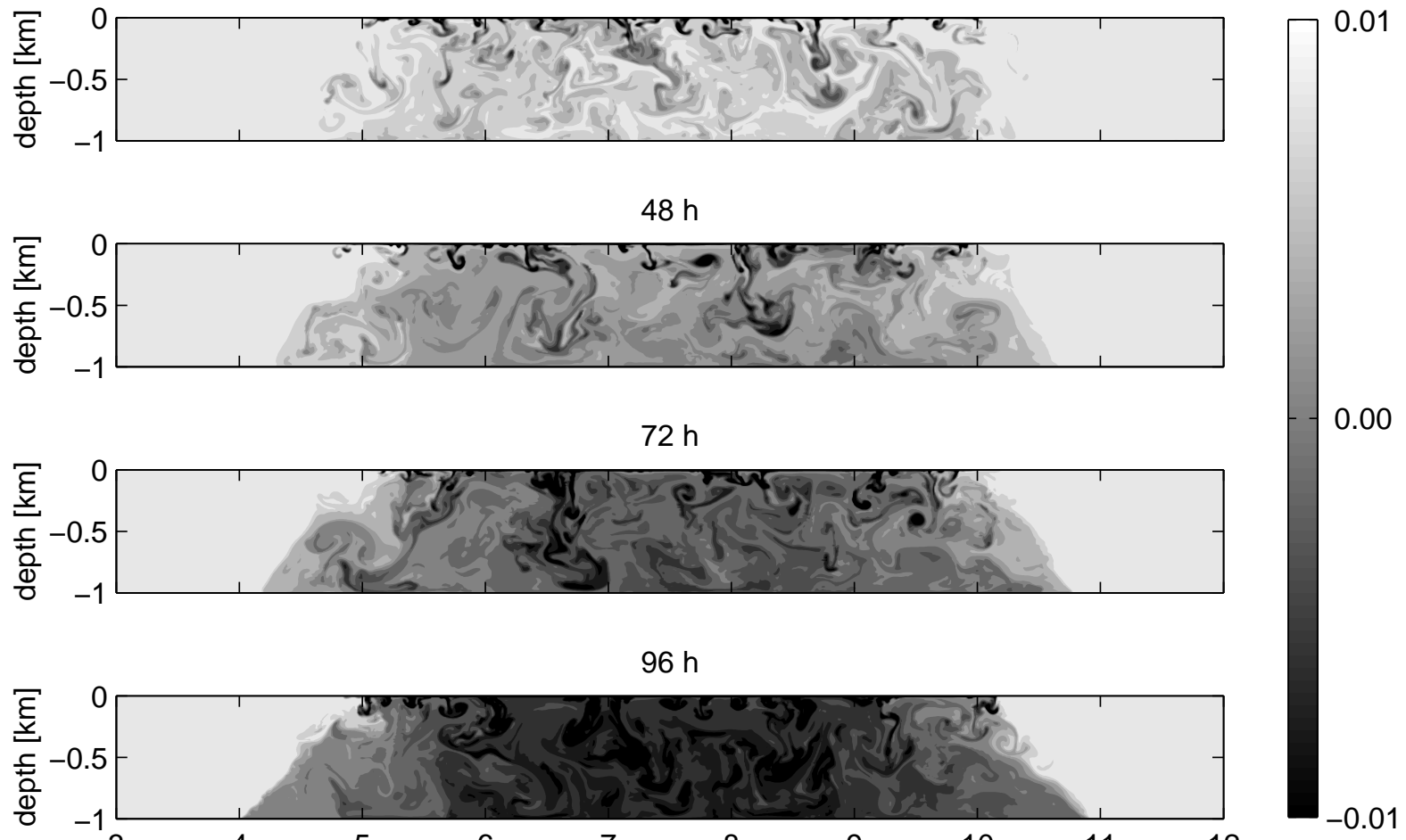

$96 \mathrm{~h}$

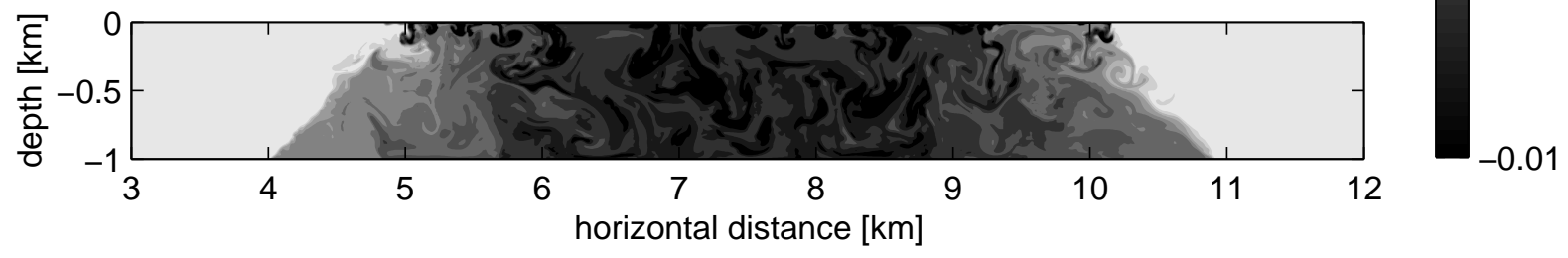

Fig. 1. Potential temperature in the convection region of the LES reference after $24,48,72$, and $96 \mathrm{~h}$ for the case of initially neutral stratification. Shading ranges form from $-0.01^{\circ} \mathrm{C}$ to $+0.01^{\circ} \mathrm{C}$, with a contour interval of $0.002^{\circ} \mathrm{C}$. Three kilometers of quiescent fluid on each side of the displayed domain are not shown.

(bottom row) can reproduce this stratification (except near the bottom boundary); both convective adjustment (CA, top row) and implicit diffusion (ID, middle row) lead to temperatures that increase with depth.

For convective adjustment, the effect of resolving the inhomogeneous surface boundary conditions is negligible (less that $0.1 \%$ ), because the equation of state is linear and the convective scheme mixes water linearly. However, as a consequence of the present implementation of convective adjustment, a coarse vertical resolution speeds up the vertical flux of cold water: in the limit of 2 vertical cells, the water column would be homogenized instantly.

For implicit diffusion (middle row) the effect of horizontal resolution is largest at the onset of convection. Also lower vertical resolution reduces the effect of horizontal resolution dramatically. With a resolution of only 18 layers, the difference between the solution with 3 horizontal grid nodes and 1 horizontal node is less than $20 \%$ and barely visible in Fig. 2 . With increasing time the vertical temperature profile becomes less dependent on resolution until after 96 hours all resolutions give virtually the same result.

The KPP solutions depend only slightly on both horizontal and vertical resolution. Only immediately after the onset of convection do the profiles differ noticeably from the reference solution (not shown).

\subsection{Continuous (linear) stratification}

We repeat the experiments of the previous section with an initial density field that varies linearly with depth: $\rho(z)=\rho_{0}\left(1-\frac{N^{2}}{g} z\right)$, equivalent to $\theta(z)=\theta_{0}+$ $\frac{N^{2}}{g \alpha} z$, with a buoyancy frequency $N^{2}=5 \times 10^{-7} \mathrm{~s}^{-1}$. This stratification is marginally stable. Fig. 3 shows 

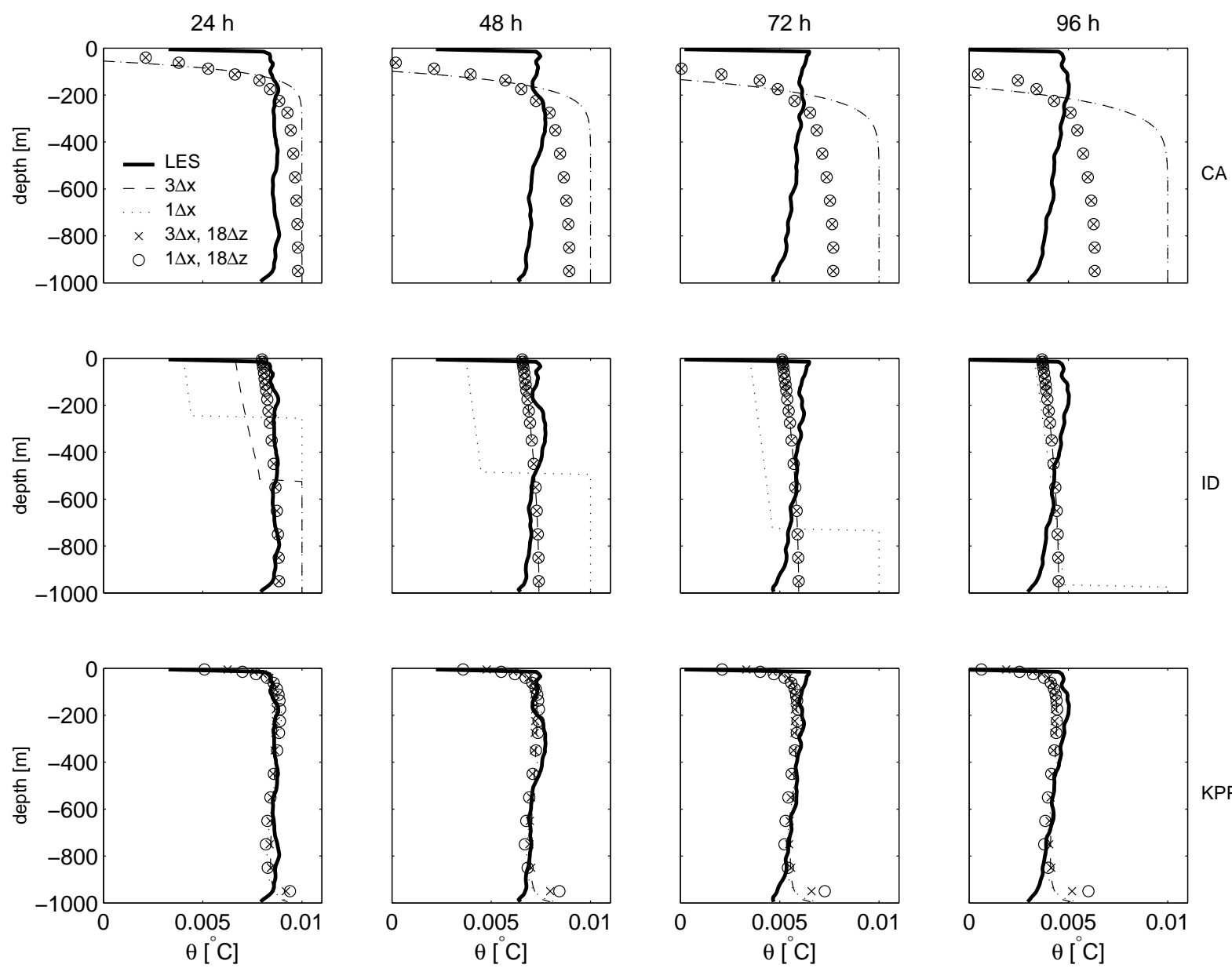

Fig. 2. Comparison of vertical profiles of the horizontally averaged temperature without initial stratification after $24,48,72$, and 96 hours of integration (from left to right). Convection is parameterized as convective adjustment (CA, top row), implicit diffusion (ID, middle row), and KPP (bottom row). The thick solid line represents the reference LES solution. The horizontal grid spacing in the parameterization experiments is $5 \mathrm{~km}$ ( 3 horizontal grid nodes, dashed lines, $3 \Delta x)$, so that the buoyancy loss is resolved by the grid, and $15 \mathrm{~km}(1 \mathrm{horizontal}$ grid node, dotted lines, $1 \Delta x$ ) in which case an average heat flux is applied. The crosses (3 horizontal grid nodes) and open circles (1 horizontal grid node) represent corresponding experiments with lower (more OGCM-like) vertical resolution of 18 layers (18 $\Delta z$ ).

the temperature profiles in the top $500 \mathrm{~m}$ of the water column. In contrast to Section 3.1, now the horizontal resolution matters: the models with only 1 horizontal grid node and averaged heat flux generally mix too strongly but not deep enough, whereas the models with 3 horizontal grid nodes reproduce the LES reference more accurately.

Convective adjustment (top row) in the present implementation and with the 1 hour time step cannot mix the cold water down fast enough, so that the water column near the surface remains unstable and the water below $100 \mathrm{~m}$ depth remains too warm even at the best resolution. On the other hand, the models with KPP and implicit diffusion are almost indistinguishable. In the $3 \Delta x$ case, they represent the LES reference much better than the model with convective adjustment.

\subsection{Density profile with pycnocline at $200 \mathrm{~m}$}

In the seasonally ice-covered regions around the Antarctic continent and in the Weddell Sea, the vertical density profile is often characterized by a homoge- 

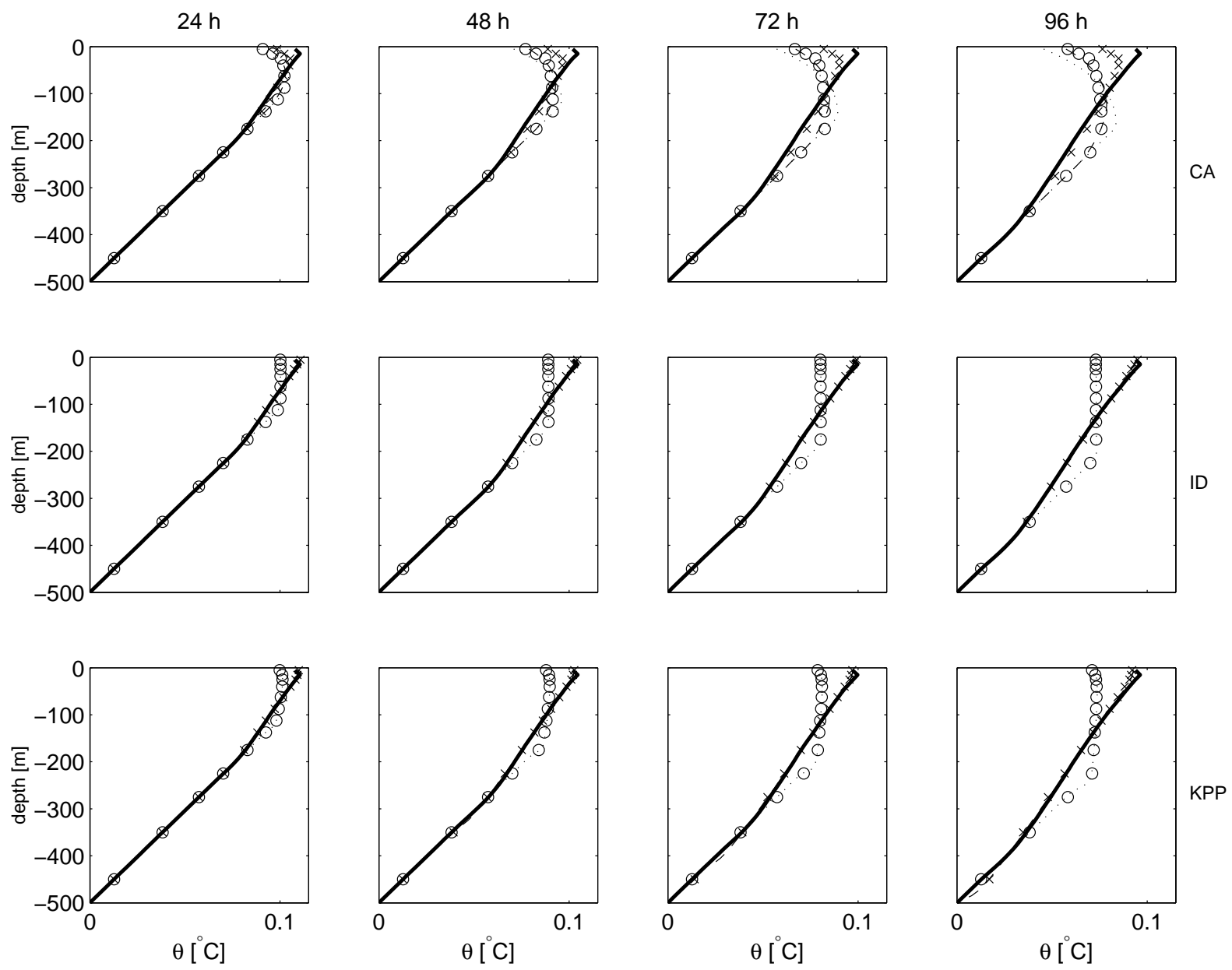

Fig. 3. Same as Fig. 2 but for linear ambient stratification. Solid line: LES solution, dashed line: $3 \Delta x$, dotted line: $1 \Delta x$, crosses: $3 \Delta x 18 \Delta z$, circles: $1 \Delta x 18 \Delta z$.

neously mixed surface layer followed by a sharp pycnocline near $200 \mathrm{~m}$ (Olbers et al., 1992). Convective plumes may penetrate into the pycnocline, further increasing the complexity of the situation. From a typical hydrographic profile, we derive an initial temperature profile from the vertical integral of the buoyancy frequency squared,

$$
\theta(z)=\theta\left(z_{0}\right)+\int_{z_{0}}^{z} \frac{N^{2}}{g \alpha} d z^{\prime},
$$

and repeat both the reference and the coarse model simulations as in the previous sections. The results are summarized in Fig. 4.

Because of the strong stratification between $70 \mathrm{~m}$ and $200 \mathrm{~m}$ depth, vertical mixing is slow and confined to the upper $200 \mathrm{~m}$. For all three parameterizations the vertical density structure can only be properly reproduced when the horizontal variation of the buoyancy loss is resolved. If there is only one horizontal grid node and the surface buoyancy loss is horizontally averaged the average temperature profile is vertically homogenized because convection mixes the water column over the entire width of the domain as in the previous section. However, this behavior emerges only after $72 \mathrm{~h}$, which is the time needed to overcome the initial stratification. As before, the models with increased vertical diffusivity and KPP yield temperature profiles that are closer to the LES reference than that of the convective adjustment scheme model. 

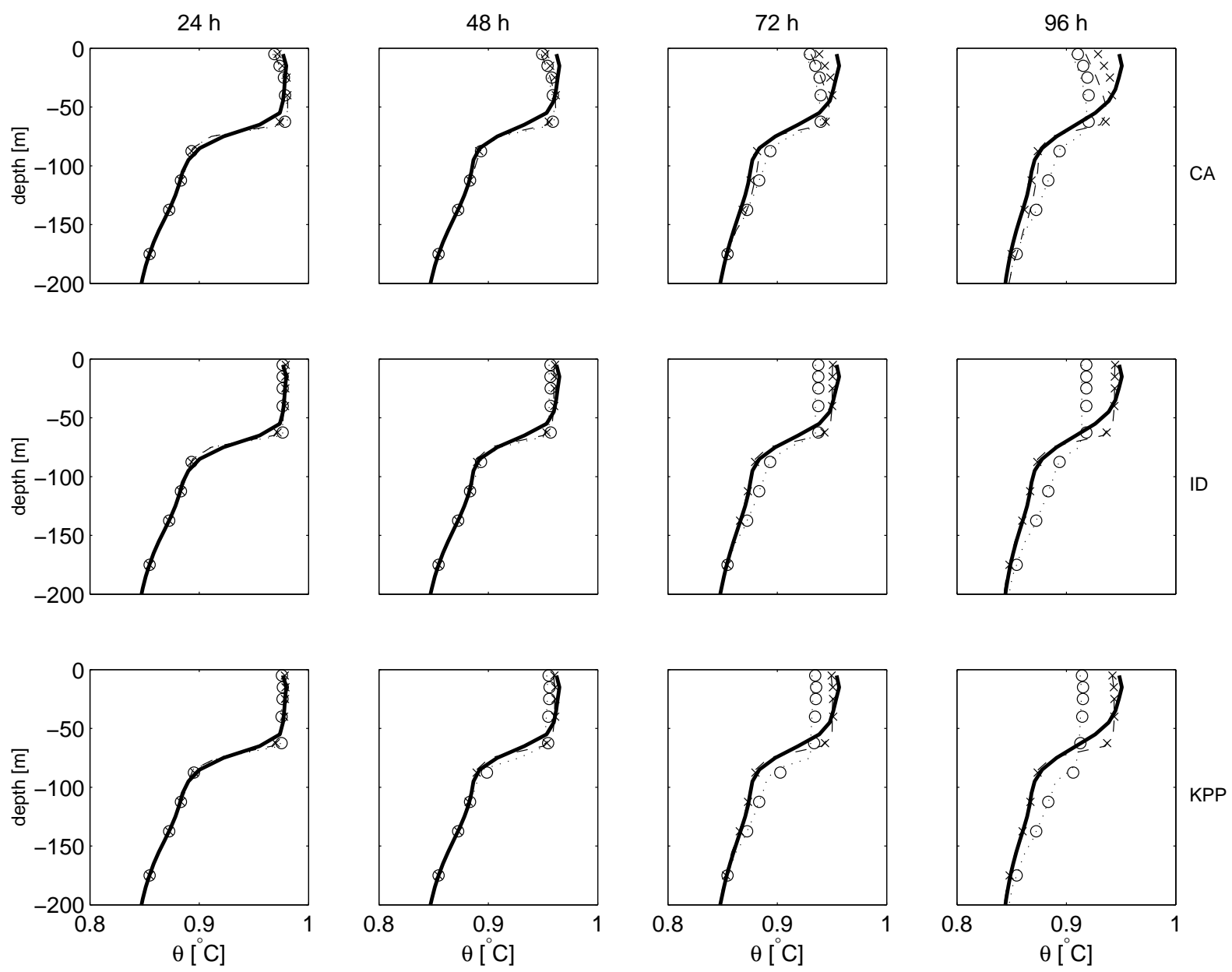

Fig. 4. Same as Fig. 2 but for an initial pycnocline. Solid line: LES solution, dashed line: $3 \Delta x$, dotted line: $1 \Delta x$, crosses: $3 \Delta x 18 \Delta z$, circles: $1 \Delta x 18 \Delta z$.

\section{Idealized Mixing with Linear Stratification}

In the limit of instantaneous vertical mixing and no horizontal mixing, we can explain the behavior in the experiments with linear stratification (Section 3.2) with simple geometrical considerations (c.f. Fig. 5). With instantaneous vertical mixing, the heat loss $Q d t$ leads to a deepening of the mixed layer, so that after a finite time $\Delta t=t-t_{0}$, the base of the mixed layer is at $z=-h(t)$. Before the cooling event, the vertical average of the potential temperature over this depth is

$$
\langle\theta\rangle=\theta_{0}-s \frac{h}{2}
$$

where $s=\frac{N^{2}}{g \alpha}$. The change of the ocean heat content is determined by the heat loss at the surface,

$$
\begin{aligned}
c_{w} \rho_{0} \int_{h(t)}^{0}\left[\theta(z)-\theta_{0}\right] d z & =\int_{t_{0}}^{t} Q d t \\
c_{w} \rho_{0} s \frac{h^{2}(t)}{2} & =Q\left(t-t_{0}\right),
\end{aligned}
$$

where $c_{w}$ is the heat capacity of water and $\theta_{0}$ a constant reference temperature. This yields an expression for the mixed layer depth:

$$
h(t)=\sqrt{\frac{2 Q\left(t-t_{0}\right)}{c_{w} \rho_{0} s}} .
$$

The new temperature in the mixed layer at time $t$ is 


$$
\theta(t)=\langle\theta\rangle-\Delta \theta=\langle\theta\rangle-\frac{Q\left(t-t_{0}\right)}{c_{w} \rho_{0} h(t)}
$$

In the case when the surface forcing is resolved, only one third of the domain is mixed in this way, while the other two remain unchanged. Taking the horizontal average yields the new temperature profile above $z=-h$ :

$$
\begin{aligned}
\theta_{\text {mixed }}(t, z)= & \frac{1}{3}\left(\theta_{0}-s \frac{h(t)}{2}-\frac{Q\left(t-t_{0}\right)}{c_{w} \rho_{0} h(t)}\right) \\
& +\frac{2}{3}\left(\theta_{0}+s z\right) \\
= & \theta_{0}-\frac{1}{3}\left(s \frac{h(t)}{2}+\frac{Q\left(t-t_{0}\right)}{c_{w} \rho_{0} h(t)}\right) \\
& +\frac{2}{3} s z .
\end{aligned}
$$

The first two terms in(6) are the new (reduced) temperature at the surface followed by a slower temperature decrease with depth (new slope $s_{\text {mixed }}=\frac{2 s}{3}$ ). Below $z=-h$, the profile is continued by the initial profile.

In the case of heterogeneous boundary conditions the entire domain is cooled by one third of the heat flux. After denoting the modified heat flux and mixing depth by stars, we write for the temperature of the homogenized mixed layer

$$
\begin{aligned}
\theta_{\text {mixed }}^{*} & =\theta_{0}-s \frac{h^{*}(t)}{2}-\frac{Q^{*}\left(t-t_{0}\right)}{c_{w} \rho_{0} h^{*}(t)} \\
& =\theta_{0}-\frac{\sqrt{3}}{3}\left(s \frac{h(t)}{2}+\frac{Q\left(t-t_{0}\right)}{c_{w} \rho_{0} h(t)}\right),
\end{aligned}
$$

because $Q^{*}=\frac{1}{3} Q$ and from (4), $h^{*}=\frac{\sqrt{3}}{3} h$. Again, below $z=-h^{*}$, the profile remains unchanged. The resulting temperature profiles are shown in Fig. 5. The shape of the profiles in Fig. 3, at least for implicit diffusion and KPP, is clearly the same as the ones derived from simple geometrical considerations, showing that those schemes mix very fast. The convective adjustment scheme in this particular implementation apparently is too slow to be considered "instantaneous".

In the case of $s=0$, that is, for neutral stratification, the mixing depth is identical to the depth of the domain for both cases $\left(h^{*}=h\right)$, and equations (6) and (7) give the same results, as confirmed by the profiles in Fig. 2 .

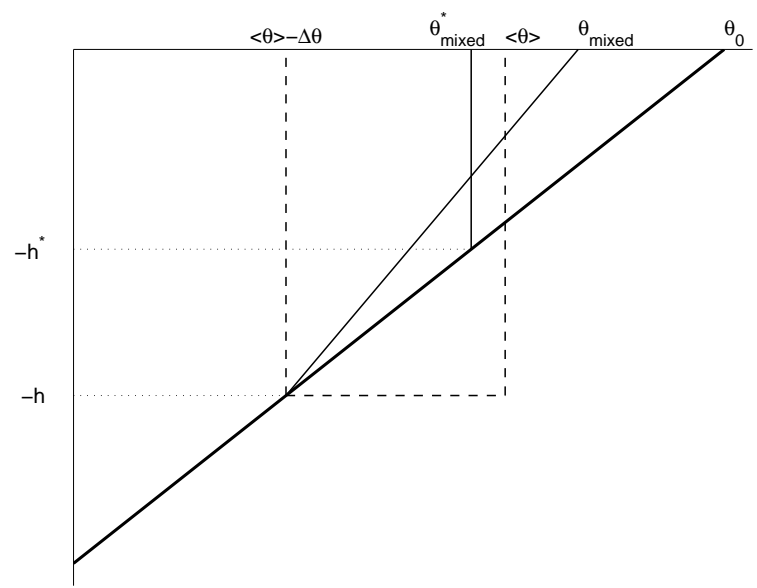

Fig. 5. Geometrical interpretation of horizontal averaging and heterogeneous surface boundary conditions. The thick solid line is the initial temperature profile; the thin solid lines are the final profiles for resolved and heterogeneous (starred variables) boundary conditions; the dashed lines denote the mean temperature before and after a cooling event if the full surface heat flux is used (c.f. Eqs. (1) and (5)).

\section{Discussion and Conclusion}

All vertical mixing schemes used in this study reproduce the averaged temperature profiles of the LES reference runs reasonably well when the spatial variation of the surface heat flux is resolved by the computational grid. On average and especially on the 1-2 day time scales, the KPP scheme outperforms mixing by increased vertical diffusivity (implicit diffusion) and convective adjustment, which is not surprising, given the higher sophistication of the KPP scheme. With a long time step of 1 hour typical for a coarse, largescale OGCM, convective adjustment in the form implemented in the MITgcm mixes the water column too slowly leading to notoriously unstable stratification. This is particularly apparent in the case of neutral and linear stratification (cf. Fig. 2 and Fig. 3) when compared to the theoretical limit of instantaneous vertical mixing (Section4). Different implementations of convective adjustment that instantly mix the entire water column down to the deepest level of instability remove instabilities more efficiently (Madec et al., 1991), but may be too fast (Timmermann and Beckmann, 2004).

The performance of the mixing schemes degrades for heterogeneous boundary conditions, that is, when the horizontal grid spacing is too big to resolve the 
surface heat flux heterogeneity. In general, horizontal averaging of the surface boundary conditions has two effects: mixing does not penetrate deep enough into the water column when compared to the LES reference; at the same time, mixing is too strong above the penetration depth and leads to vertically homogenized temperature profiles, with too cold water near the surface and too warm water below the penetration depth. We have shown that a simple model can explain the effect of heterogeneous boundary conditions for simple density profiles in the limit of instantaneous vertical mixing as a result of horizontal averaging.

An exception is the model with the KPP scheme in the case of initially neutral stratification. In this case the model can reproduce the vertical structure of the reference run, in particular the stable stratification, independently of resolution and integration time. This behavior can be explained as follows: In the LES reference solution, the convective plumes, driven by the horizontal pressure gradients, are dispersed laterally after they have reached the bottom of the domain. Thereby, the area of cold fluid is extended into regions of no convection so that in those regions a stable profile emerges as cold dense fluid moves beneath warm light fluid (Fig. 1), resulting in the horizontally averaged profile in Fig. 2. KPP produces this type of profile with dense fluid beneath lighter fluid by means of the nonlocal transport term (Large et al., 1994). This term is determined mostly from surface fluxes and therefore, in our case of heat loss at the surface, it represents an additional upward heat flux over the entire water column, which is not present in the implicit diffusion scheme. Consequently, even after $96 \mathrm{~h}$ the implicit diffusion scheme has not removed the instability of the water column (Turning off the nonlocal transport term in KPP leads to an instable profile for KPP that is similar to the implicit diffusion case, not shown). In the present case, KPP is independent of the horizontal resolution because the nonlocal transport scales roughly linearly with the surface flux.

In all experiments the surface buoyancy loss was prescribed and constant. In more realistic situations with sensible and latent heat fluxes and fresh water fluxes (brine rejection) during sea-ice formation and melting, the impact of heterogeneous boundary conditions or horizontal resolution may be larger than in our idealized conditions. High frequency surface forcing may result in poorly modeled density profiles, es- pecially for the simpler schemes, as they do not react to the surface fluxes as quickly as KPP does. Further, using the full equation of state introduces more non-linearities into the system which may also lead to larger effects of heterogeneous boundary conditions. Horizontal mixing has been neglected in this study because of the short time scales that were considered. For longer times scales effects such as baroclinic instabilities and vortex spreading during the breakup of convective patches (Marshall and Schott, 1999) will modify our results. The presence of a strong horizontal flow might have a similar effect.

We conclude that, if horizontal resolution is not sufficient to resolve the spatial patterns of the surface buoyancy flux, for example in the presence of seaice with concentrations smaller than $100 \%$, heterogeneous boundary conditions need to be taken into account to accurately model the vertical density structure and deep water formation in coarse resolution models. In the simplest case, this may be done in a way that is similar to the ocean plume parameterization scheme (OPPS, Paluszkiewicz and Romea, 1997): In the case of heterogeneous boundaries, stability and vertical mixing could be computed for two different density classes (i.e., two different temperatures and salinities) per grid cell. In this way, more realistic density profiles may be obtained in coarse grid simulations at relatively low cost.

On the other hand, sea-ice models generally only provide the fractional ice cover (ice-concentration) as a variable. However, many different sea-ice distributions can yield the same ice-concentration value. The extreme cases are either one large ice floe or many very small ones. Because of different horizontal scales, these different conditions imply different horizontal mixing; for example, in the former case with only two separate areas one would have very little horizontal homogenization so that horizontal averaging as in Section 4 might provide the wrong starting point for the next time step. It may be necessary to maintain separate density classes over many timesteps, thus increasing storage requirements and the overall complexity of the scheme. The development of such a scheme is the subject of ongoing work. 


\section{Acknowledgements}

ML would like to thank Timo Vihma for initiating this study. We thank Sergey Danilov, Vladimir Gryanik, and Jill Schwarz for many suggestions.

\section{References}

Fernando, J. S., Chen, R., Boyer, D. L., 1991. Effects of rotation on convective turbulence. J. Fluid Mech. 228, 513-547.

Foster, T. D., 1972. Haline convection in leads and polynyas. J. Phys. Oceanogr. 2 (4), 462-469.

Gaspar, P., Grégoris, Y., Lefevre, J.-M., 1990. A simple eddy kinetic energy model for simulations of the oceanic vertical mixing: Test at Station Papa and Long-Term Upper Ocean Study Site. J. Geophys. Res. 95 (C9), 16,179-16,193.

Golitsyn, G. S., 1980. Geostrophic convection (in Russian). Dokl. Akad. Nauk. SSSR 251, 1356-1360.

Haidvogel, D. B., Beckmann, A., 1999. Numerical Ocean Circulation Modelling. Vol. 2 of Series on Environmental Science and Management. Imperial College Press, London.

Hundsdorfer, W., Koren, B., van Loon, M., Verwer, J., 1995. A positive finite-difference advection scheme. J. Comp. Phys. 117 (1), 35-46.

Hundsdorfer, W., Trompert, R. A., 1994. Method of lines and direct discretization: a comparison for linear advection. Applied Numerical Mathematics 13 (6), 469-490, doi:10.1016/01689274(94)90009-4.

Jones, H. L., Marshall, J. C., 1993. Convection with rotation in a neutral ocean: A study of open-ocean deep convection. J. Phys. Oceanogr. 23 (6), 10091039.

Krauss, E. B., Turner, J. S., 1967. A one-dimensional model of the seasonal thermocline. II: The general theory and its consequences. Tellus 19, 98-105.

Large, W. G., McWilliams, J. C., Doney, S. C., 1994. Oceanic vertical mixing: A review and a model with a nonlocal boundary layer parameterization. Rev. Geophys. 32 (4), 363-404.

Leith, C. E., 1968a. Diffusion approximation for turbulent scalar fields. Phys. Fluids 11 (8), 1612-1617.

Leith, C. E., 1968b. Diffusion approximation for two- dimensional turbulence. Phys. Fluids 11 (3), 671673.

Madec, G., Delecluse, P., Crepon, M., Chartier, M., 1991. A three-dimensional numerical study of deepwater formation in the Northwestern Mediterranean Sea. J. Phys. Oceanogr. 21 (9), 1349-1371.

Marshall, J., Adcroft, A., Hill, C., Perelman, L., Heisey, C., 1997. A finite-volume, incompressible Navier Stokes model for studies of the ocean on parallel computers. J. Geophys. Res. 102 (C3), 5753 5766.

Marshall, J., Schott, F., 1999. Open-ocean convection: Observations, theory, and models. Rev. Geophys. 37 (1), 1-64.

Mellor, G. L., Yamada, T., 1982. Development of a turbulence closure model for geophysical fluid problems. Rev. Geophys. Space Phys. 20, 852-875.

MITgcm Group, 2002. MITgcm Release 1 Manual. (online documentation), MIT/EAPS, Cambridge, MA 02139, USA, http://mitgcm.org/ sealion/online_documents/manual. html.

Mosby, H. A., 1934. The waters of the Atlantic Antarctic Ocean. Scientific Research of the Norwegian Antarctic Expeditions 1927-1928 1, 1-131.

Olbers, D., Gouretski, V., Seiss, G., Schröter, J., 1992. Hydrographic Atlas of the Southern Ocean. AlfredWegener-Institut für Polar- und Meeresforschung.

Pacanowski, R. C., Philander, S. G. H., 1981. Parameterization of vertical mixing in numerical models of the tropical ocean. J. Phys. Oceanogr. 11, 14431451.

Paluszkiewicz, T., Romea, R. D., 1997. A onedimensional model for the parameterization of deep convection in the ocean. Dyn. Atmos. Oceans 26, 95-130.

Timmermann, R., Beckmann, A., 2004. Parameterization of vertical mixing in the Weddell Sea. Ocean Modelling 6 (1), 83-100.

Timmermann, R., Beckmann, A., Hellmer, H. H., 2002. Simulation of ice-ocean dynamics in the Weddell Sea. Part I: Model configuration and validation. J. Geophys. Res. 107 (C3), 3024, doi:10.1029/2000JC000741. 